\title{
Clinicopathological and immunohistochemical features of pulmonary artery sarcoma: A report of three cases and review of the literature
}

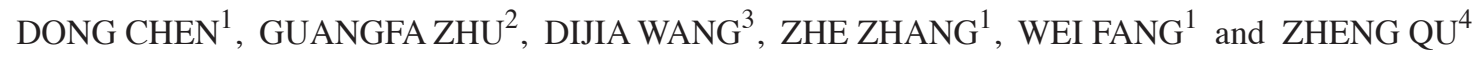 \\ Departments of ${ }^{1}$ Pathology, and ${ }^{2}$ Respiratory and Critical Care Medicine, Beijing Anzhen Hospital, \\ Capital Medical University, Beijing Institute of Heart, Lung and Blood Vessel Diseases, Beijing 100029; \\ ${ }^{3}$ Department of Intensive Care Medicine, Beijing Tongren Hospital, Capital Medical University, Beijing 100730; \\ ${ }^{4}$ Department of Cardiac Surgery, Beijing Anzhen Hospital, Capital Medical University, \\ Beijing Institute of Heart, Lung and Blood Vessel Diseases, Beijing 100029, P.R. China
}

Received February 15, 2015; Accepted January 4, 2016

DOI: $10.3892 / 01.2016 .4308$

\begin{abstract}
Pulmonary artery sarcoma (PAS) is an extremely rare and highly malignant tumor that originates in the pulmonary artery. The majority of reported cases of PAS are confirmed by pathological examination subsequent to surgery or by autopsy. The present study reports the clinicopathological characteristics and immunohistochemical phenotypes of three cases of PAS, and aims to facilitate the identification of this lethal disease. In the present study, the data from clinical, histopathological and immunohistochemical examinations of three patients with PAS, whose diagnoses were confirmed by surgical biopsy conducted at the Beijing Anzhen Hospital (Beijing, China) between 2008 and 2012, were retrospectively analyzed. The patients (two women and one man; average age, 41.3 years old) presented with dyspnea on exertion. In addition, two of the patients experienced chest tightness, and one patient experienced intermittent syncope. Computed tomography pulmonary angiography revealed that two of the patients possessed a filling defect in the main, left and right pulmonary arteries, and one patient possessed a filling defect in the right upper pulmonary artery. Macroscopically, the PAS appeared as a mucoid intraluminal or nodular sessile mass spreading along the pulmonary artery. Microscopically, the tumor consisted of spindle cells with fascicular and storiform patterns, and was accompanied by necrosis and stromal myxoid changes. Immunohistochemically, vimentin, desmin and cluster of differentiation 34 were highly expressed in the patient that was
\end{abstract}

Correspondence to: Dr Guangfa Zhu, Department of Respiratory and Critical Care Medicine, Beijing Anzhen Hospital, Capital Medical University, Beijing Institute of Heart, Lung and Blood Vessel Diseases, 2 Anzhen Road, Chaoyang, Beijing 100029, P.R. China

E-mail: guangfazhucn@126.com

Key words: pulmonary artery sarcoma, immunohistochemistry, histology, differential diagnosis diagnosed with intimal sarcoma, while vimentin and $\alpha$-smooth muscle actin were highly expressed in the other two patients, who were diagnosed with leiomyosarcoma. PAS is often misdiagnosed due to nonspecific clinical manifestations and radiological features. Therefore, the diagnosis of PAS should be based on typical morphological features and immunohistochemical analysis of the tumor tissue.

\section{Introduction}

Pulmonary artery sarcoma (PAS) is an extremely rare and highly malignant tumor that originates in the pulmonary artery (1). It has an incidence rate of $0.001-0.030 \%$ (2). Since 1923, when the first case of PAS was reported by Mandelstamm (3), $\leq 300$ cases have been reported to date worldwide (4-7). The etiology of PAS is unknown, and this disease has a poor prognosis (5). Early diagnosis followed by radical surgical resection constitutes the only chance of survival for patients with PAS (5), and patients have an average survival time of $\sim 1.5$ months without surgical treatment (8). However, due to the rare and nonspecific clinical manifestations and imaging findings, PAS is frequently misdiagnosed as various pulmonary thromboembolic diseases, including pulmonary thromboembolism (PTE) or chronic thromboembolic pulmonary hypertension (CTEPH), and the majority of reported PAS cases are confirmed by pathological examination subsequent to surgery or by autopsy $(2,9,10)$. The present study reports three cases of PAS that were initially misdiagnosed as PTE or CTEPH, and were later identified as PAS following surgery. The clinicopathological and immunohistochemical characteristics of these cases are reported in the present study to aid the understanding and differential diagnosis of the PAS tumor in clinical practice.

\section{Case report}

Between 2008 and 2012, three adult patients with PAS, whose diagnoses were confirmed by surgery and pathological examinations, were managed by the Beijing Anzhen Hospital (Beijing, 

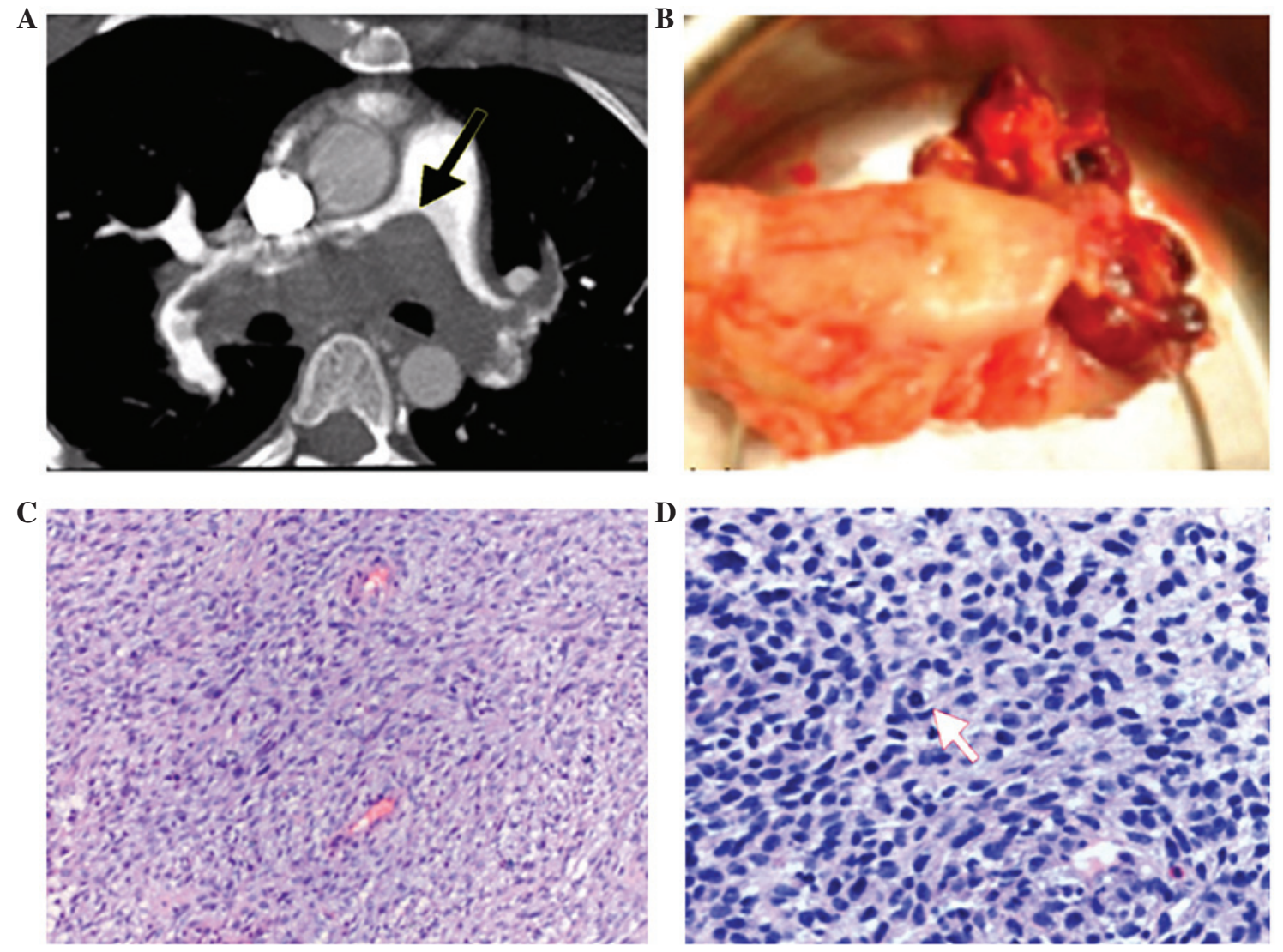

Figure 1. Case 1. (A) Computed tomography pulmonary angiography reveals that the lumen of the pulmonary trunk is obliterated by a low-density mass (black arrow), which extends into the left and right pulmonary arteries. (B) Macroscopic findings show a smooth gelatinous tumor of $9.5 x 4.5$ cm in size. (C and D) Microscopic findings reveal an abundance of spindle cells with pathological mitosis (white arrow). Hematoxylin and eosin staining; magnification, (C) $\times 100$ and (D) $\times 200$.

China). The characteristics of each patient are summarized in Table I. The patients, two women and one man with a mean age of 41.3 years old (range, 36-47 years), possessed disease histories that ranged between 50 days and 4 years. All the patients experienced dyspnea due to exertion, and two of them also experienced chest tightness, while one patient experienced repeated and intermittent syncope. The electrocardiogram (ECG; ECG-903; Shenzhen Spring Technology Industry Co., Ltd., Shenzhen, China) revealed that one patient possessed right ventricular enlargement, in addition to ST segment and T wave changes (case 1); another patient exhibited SI QIII TIII pattern (case 2); and the other patient possessed a normal ECG (case 3). Echocardiography (Sonos 2500 Ultrasound; Hewlett-Packard, Palo Alto, CA, USA) showed that all the patients presented pulmonary artery expansion, pulmonary artery hypertension and right ventricular hypertrophy, and two of the patients (cases 1 and 3) were suggested to possess PTE. The chest radiographs (MobileDaRt Evolution; Shimadzu Corporation, Kyoto, Japan) revealed a thickening of the right or left pulmonary artery in two patients (cases 2 and 3), and normal findings in one patient (case 1). Computed tomography pulmonary angiography (CTPA; Aquilion ONE; Toshiba Medical Systems Corporation, Otawara, Japan) revealed that two of the patients presented a filling defect in the main, left and right pulmonary arteries, and were diagnosed as central PTE (cases 1 and 3; case 1, Fig. 1A), while the third patient presented expansion of the main pulmonary artery and left pulmonary artery lumen, and a filling defect in the right upper pulmonary artery (case 2). The lung ventilation/perfusion (V/Q) scan (Technegas Generator; Cyclomedica Australia, Pty, Ltd., Lucas Heights, NSW, Australia) of one patient revealed that all the segments of the left lung and the right upper lung lobe possessed perfusion defects, but the ventilation was normal (case 1), whereas another patient presented diffuse lung V/Q mismatch (case 3). The dual-color Doppler ultrasound examination (iE33 Ultrasound Machine; Philips Medical Systems, Inc., Bothell, WA, USA) revealed no abnormalities in the deep veins of the legs of all three patients. Clinically, two of the patients were diagnosed with CTEPH (cases 1 and 2), while one patient was diagnosed with PTE (case 3). Pulmonary artery thromboendarterectomies were performed for the patients diagnosed with CTEPH (cases 1 and 2), while an embolectomy was performed for the patient diagnosed with PTE (case 3). During the surgery, tumor tissues were identified in the main, left and right pulmonary arteries. Lesions were attached to the vessel wall, grew into the lumen, and were shaped along the vessel branches in two of the patients (cases 1 and 3), whereas lesions were located in the right pulmonary artery in one patient (case 2). The tumor tissues were adhesive to the vessel wall, and not easily separated from it. Surgery was attempted to maximally remove the tumor tissue in all the patients.

Macroscopic examination of the tumor specimens (Leica DM3000; Leica Microsystems, Inc., Buffalo Grove, IL, USA; 


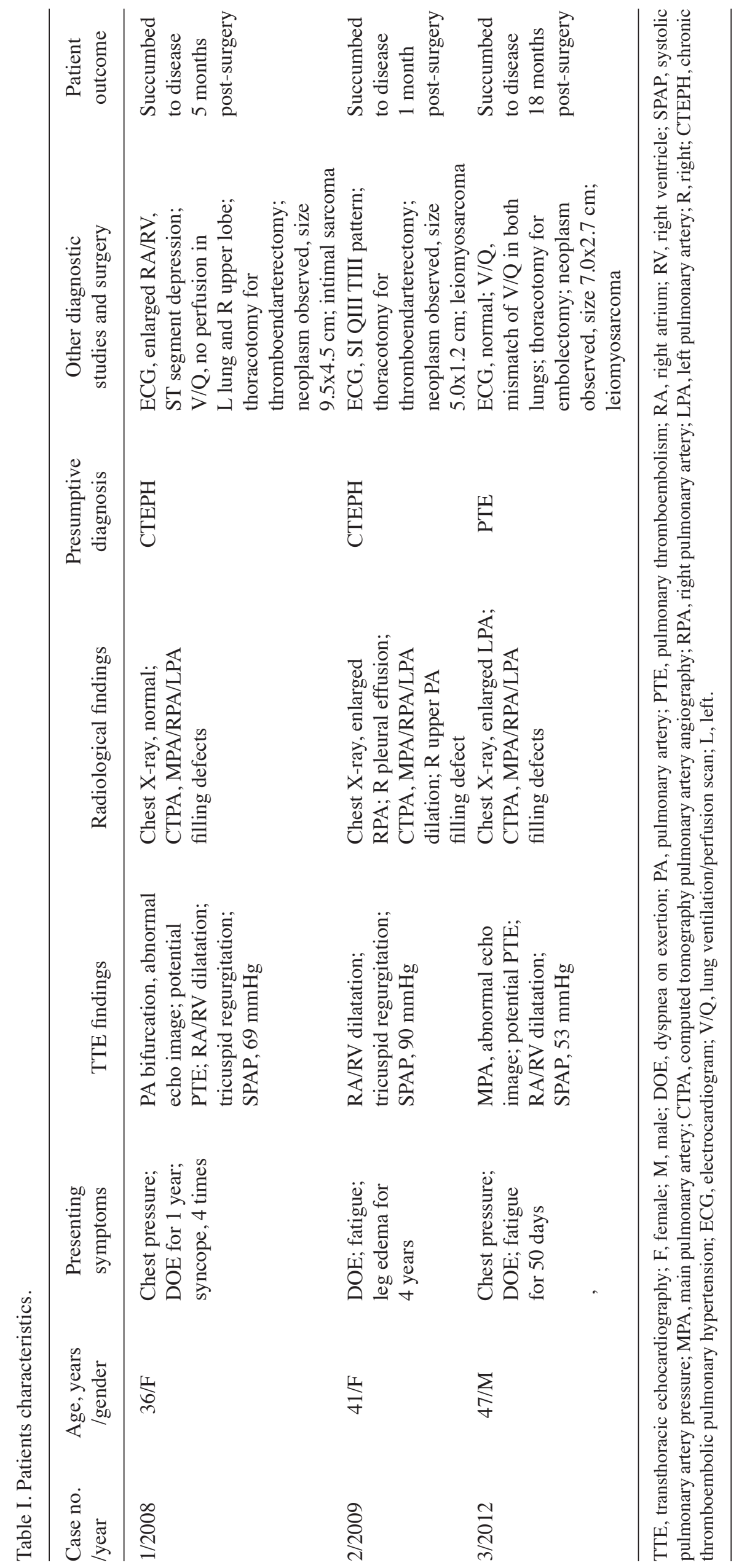


A

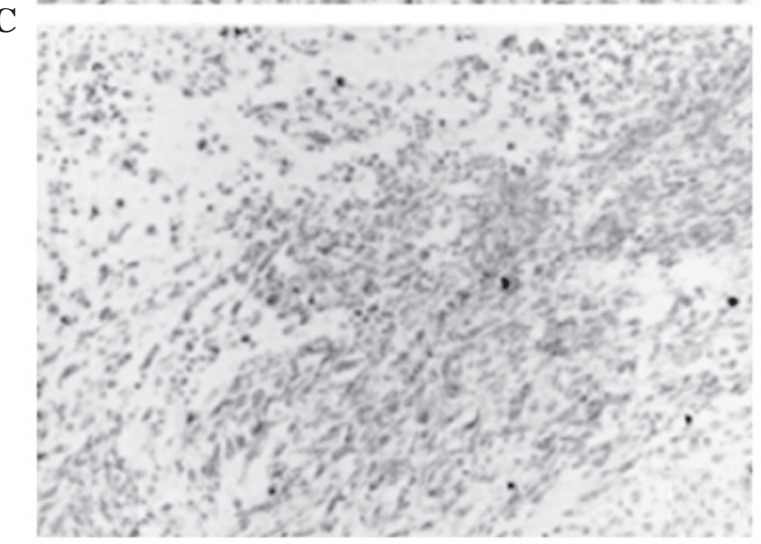

B

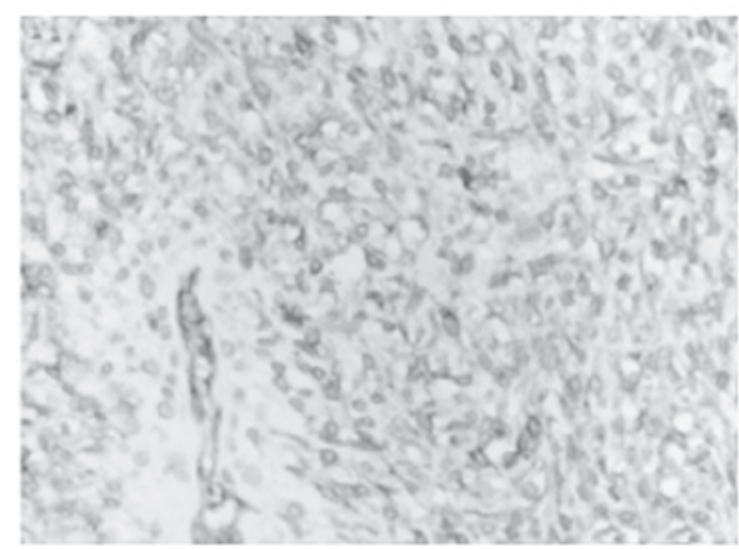

D

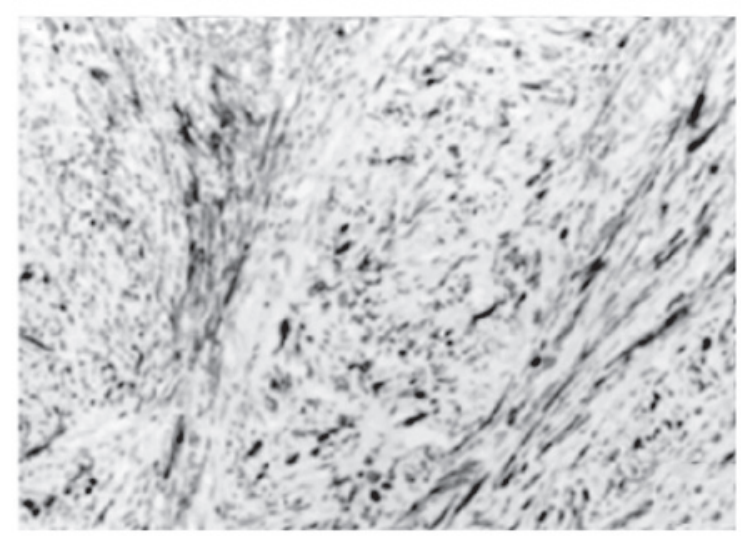

Figure 2. Case 1. Immunohistochemical staining using Dako EnVision Systems reveals that (A) vimentin, (B) cluster of differentiation 34, (C) desmin and (D) $\alpha$-smooth muscle actin are expressed in the tumor cells.

camera: AxioCam MRc5, Zeiss AG, Oberkochen, Germany) revealed that two of the patients presented mucoid or smooth gelatinous tumor tissue that filled the vascular lumens. Certain regions appeared to be partially wrapped by a capsule. The tumor surface possessed firm fibrotic regions with gray or brown cut surface. The sizes of the tumors were $9.5 \times 4.5 \mathrm{~cm}$ (case 1, Fig. 1B) and $7.0 \times 2.7 \mathrm{~cm}$ (case 3). The tumor of the patient corresponding to case 2 was $5.0 \times 1.2 \mathrm{~cm}$ in size, and possessed broken and gray soft tissue with a crisp texture.

The resected tumors were subjected to histological and immunohistochemical analysis. For that purpose, specimens were fixed in 4\% neutral formalin (Beijing Yili Fine Chemical Co., Ltd., Beijing, China), embedded in paraffin (Beijing Yili Fine Chemical Co., Ltd.), sectioned, and stained with hematoxylin and eosin (Beijing Yili Fine Chemical Co., Ltd.). Dako EnVision System (Agilent Technologies, Inc., Santa Clara, CA, USA) $(11,12)$ was used for immunohistochemical analysis. The following antibodies were used, which were all mouse monoclonal anti-human, unless otherwise specified, diluted in phosphate-buffered saline and purchased from Beijing Zhongshan Golden Bridge Biotechnology Co., Ltd. (OriGene Technologies, Inc., Beijing, China): Vimentin (clone, V9; catalog no., ZM-0260; dilution, 1:150), desmin (clone, D33; catalog no., ZM-0091; dilution, 1:100), cluster of differentiation (CD)34 (clone, QBEnd/10; catalog no., ZM-0046; dilution, 1:150), CD31 (clone, 1A10; catalog no., ZM-0044; dilution, 1:100), $\alpha$-smooth muscle actin ( $\alpha$-SMA; clone, HHF35; catalog no., ZM-0001; dilution, 1:150), cytokeratin (clone, AE1/AE3; catalog no., ZM-0069; dilution, 1:150), epithelial membrane antigen (EMA; clone, GP1.4; catalog no., ZM-0095; dilution, 1:150), CD68 (clone, 514H12; catalog no., ZM-0060; dilution, 1:150), CD117 (clone, 2E4; catalog no., ZM-0437; dilution, 1:100), S-100 (catalog no., ZA-0225; dilution, 1:150), human melanoma black 45 (clone, HMB45; catalog no., ZM-0187; dilution, 1:150), myelin basic protein (MBP; clone, 7H11; catalog no., ZM-0488; dilution, 1:200), myogenic differentiation 1 (MyoD1; rabbit anti-human monoclonal; clone, Ep212; catalog no., ZA-0585; dilution, 1:150), myosin (clone, MY32; catalog no., ZM-0196; dilution, 1:150) and myoglobin (clone, MYO18; catalog no., ZM-0193; dilution, 1:150). The immunohistochemical assays were performed according to the manufacturer's protocol with an incubation time of $30 \mathrm{~min}$ at $25^{\circ} \mathrm{C}$. Pathological diagnoses were established based on the 2004 and 2002 'World Health Organization Classification of Tumors' $(1,13)$.

The histopathological and immunohistochemical results revealed that the tumor of case 1 possessed a large number of spindle cells arranged as bundles and braids or irregularly (Fig. 1C). Scattered necrosis and significant atypia of the tumor cells, which were mostly spindle shaped (like fibroblasts or myofibroblasts), were observed. In certain regions, the cells were differentiated epithelioid cells, with large, rod-shaped, round, oval or irregular nuclei. The chromatin was blocky and coarse. Certain cells contained 1-2 nucleoli. Mitosis was commonly identified in giant tumor cells at $\sim 20 / 10$ high power fields (Fig. 1D). Vimentin (Fig. 2A), CD34 (Fig. 2B), desmin (Fig. 2C) and $\alpha$-SMA (Fig. 2D) were expressed, while CD31, cytokeratin AE1/AE3, EMA, CD68, CD117, S-100, HMB-45, 
MBP, MyoD1, myosin and myoglobin were not. These findings were in agreement with the characteristics of intimal sarcoma (13). In two of the patients (cases 2 and 3), the tumors were composed of spindle cells arranged as interwoven bundles, and exhibited high density and compactness. Part of the tumor was mucus. The tumor cells were long and spindle-like, and contained rich cytoplasm and oblong, blunt-ended nuclei. In poorly differentiated cells, marked staining revealed that the size varied, and the nuclei were spindle-shaped and pleomorphic. The cytoplasm was eosinophilic and displayed no evident pattern. Vimentin and $\alpha$-SMA were expressed, while all other marker proteins were not expressed, with the exception of desmin and CD34 in case 3. The characteristics presented by case 2 and 3 were in agreement with a diagnosis of leiomyosarcoma (13).

Following surgery, one of the patients (case 2) died in the hospital a month later, due to septic shock and heart and lung failure. The other two patients (cases 1 and 3) were discharged from hospital, and one of them received chemotherapy (case 3). However, the two patients succumbed to tumor recurrence and metastasis 5 and 18 months subsequent to surgery, respectively.

The present study was conducted in accordance with the declaration of Helsinki, and with the approval of the Ethics Committee of Capital Medical University (Beijing, China; approval no., 2008002X). Written informed consent was obtained from all participants.

\section{Discussion}

PAS almost invariably arises from the main pulmonary arteries or the pulmonary valve $(5,14)$. When diagnosing PAS, the first step is to rule out the possibility of a metastatic tumor from other regions of the body (11). PAS is a rare malignant tumor with an incidence of $0.001-0.030 \%$, and the pathogenesis of the disease remains unclear (2). PAS has been previously reported in patients aged 13-86 years, with a mean age of 52 years old, and a female-to-male ratio of 2:1 (9). The three patients reported in the present study are consistent with the previously reported age and gender characteristics of the disease (15). PAS generally occurs insidiously (15). A tumor that protrudes toward the pulmonary artery lumen may cause the reduction of pulmonary blood volume and pulmonary hypertension (16). The most common clinical manifestation of PAS is dyspnea, which is usually accompanied by chest pain, cough, hemoptysis, fatigue and weight loss (8). A serious obstruction occurring in the pulmonary artery may result in decreased cardiac output, leading to syncope and sudden mortality (9). During physical examination, the most common signs of PAS are pulmonary valve noise, a systolic ejection murmur with a loud pulmonary component of the second heart sound (P2), jugular venous distension, hepatomegaly and peripheral edema (8).

Imaging examinations are the major tools for the diagnosis and differential diagnosis of PAS (17). Echocardiography of the large pulmonary artery and right ventricular outflow tract may reveal projections with irregular surfaces as spot echoes and hyperechoic regions. However, distinguishing PAS from pulmonary thromboembolic diseases such as PTE or CTEPH is challenging (18). Chest X-ray examinations of patients with PAS are often nonspecific, and may reveal a hilar mass, protruding pulmonary artery segment, unilateral pulmonary artery, proximal branch expansion, lung nodules, right ventricular enlargement and sparse peripheral vasculature $(8,19)$. CTPA and pulmonary magnetic resonance imaging (MRI) present advantages for the diagnosis of PAS $(7,18,20)$. CTPA may clearly reveal the involvement of lesions in the pulmonary artery, right ventricular outflow tract and pulmonary valve (17). A lesion with a full, bulged or lobulated-edged surface may be indicative of PAS (17). An MRI scan may reveal signals from the abnormal soft tissue in the main pulmonary artery cavity, but these signals are difficult to distinguish from those caused by pulmonary thromboembolic diseases (17). However, the intensity of contrast enhancement following contrast injection is associated with the degree of tumor differentiation, which is a good indicator of PAS or other malignant lesions (18). The three patients with PAS reported in the present study presented with symptoms and clinical manifestations similar to those caused by PTE, since the tumors grew inside the pulmonary artery lumen, leading to pulmonary artery stenosis or obstruction. Although the clinical and imaging data were suggestive of PAS, the data were nonspecific. As a result, all three patients were clinically diagnosed with PTE or CTEPH and referred for surgery.

According to the 2004 'World Health Organization Classification of Tumours' (1), PAS is divided into two types: Intimal and intramural. Intimal sarcoma presents an intraluminal polypoid growth pattern, and usually exhibits fibroblastic or myofibroblastic differentiation (21). Intramural sarcoma is considered distinct from intimal sarcoma, and is classified separately according to the histological subtypes as in soft tissue sarcoma (leiomyosarcoma) (4). Since intimal PAS is more common than intramural PAS, PAS is often referred to as intimal sarcoma (4). PAS often occurs in the dorsal region of the main pulmonary artery, mostly with a polypoid or finger-like form, an extends to the bifurcation of the main pulmonary artery, and the left and right pulmonary arteries (22). Certain tumors that involve the pulmonary valve may also simultaneously involve or spread to the outflow tract of the right ventricle (3). In total, $90 \%$ of PAS cases involve $\geq 2$ regions, and $85 \%$ of patients with PAS possess main pulmonary artery lesions, while $71 \%$ possess right pulmonary artery lesions, $65 \%$ possess left pulmonary artery lesions, $32 \%$ possess lesions invading the pulmonary valve and $10 \%$ possess lesions invading the outflow tract of right ventricle (4). An intimal sarcoma resembles mucoid or gelatinous clots that fill vascular lumens, and the distal extension of the tumor may possess firm fibrotic regions, be bony or gritty, and chondromyxoid foci may be present in mural lesions (23). Hemorrhage and necrosis are common in high-grade tumors $(1,13)$. The patients in the present study underwent an embolectomy or thromboendarterectomy due to the diagnosis as PTE or CTEPH. During the surgery, large emboli were identified, which closely connected to the base of the pulmonary valve or the bifurcation of the trunk and branch of the pulmonary artery. The emboli extended to the pulmonary artery in the same direction as the pulmonary vascular tree, and accounted for almost all the lumen. Gross examination revealed that the emboli were gray and brown or myxoid, but was unable to distinguish the emboli from thrombi. 
Regarding the pathogenesis and origin of PAS, the majority of studies published thus far hypothesize that an intimal sarcoma may arise from the pluripotent mesenchymal cells of the intima artery, that is, the inner membrane of the pulmonary artery (24). Therefore, PAS may have a variety of pathological types. Cox et al (4) reported the pathological results of 138 cases of PAS, of which, $43(\sim 1 / 3)$ were undifferentiated sarcoma. The second highest number of cases of PAS reported by the authors was leiomyosarcoma, followed by spindle cell sarcoma, malignant fibrous histiocytoma, fibromyxoid sarcoma, rhabdomyosarcoma, chondrosarcoma, mesenchymal tumors and osteosarcoma. According to previous immunohistochemistry and electron microscopy studies, PAS also presents other pathological types, including angiosarcoma and epithelioid hemangioendothelioma (23). Therefore, PAS has multiple histological types and may originate from pluripotent cells. The typical histological features and immunohistochemical staining for PAS are important for classifying the different pathological types (4). Under the microscope, intimal sarcoma exhibits proliferation of spindle cells in a myxoid background, alternating with hypocellular collagenized stroma $(25,26)$. Recanalized thrombi may be intimately admixed, and considerable nuclear pleomorphisms and varying degrees of spindle cells are usually present in the tumor tissue (27). The tumor may also be associated with large regional myxoid tissues and local necrosis, and typical spindle cells are arranged like a woven mat or striated, as in leiomyosarcoma. A number of intimal sarcomas and the majority of intramural sarcomas possess foci of more differentiated sarcomas, including rhabdomyosarcoma, osteosarcoma or angiosarcoma (27). In numerous intimal sarcomas, the immunohistochemical and ultrastructural examinations reveal the existence of fibroblast cells, in which vimentin is diffusely expressed, and osteopontin and $\alpha$-SMA may also be expressed (28). In a tumor that shows evidence of differentiation from vascular or smooth muscle cells, desmin and endothelial markers such as CD31, CD34 or factor VIII (FVIII) may be expressed (13). For the patient with pulmonary artery intimal sarcoma of the present study (case 1), immunophenotyping revealed that vimentin, desmin and CD34 were expressed by the tumor cells, while $\alpha$-SMA was focally expressed. These results suggest that the patient possessed PAS that exhibited differentiation into angiosarcoma and leiomyosarcoma. In previous studies, pulmonary leiomyosarcoma was also categorized as a common type of PAS, accounting for $20 \%$ of PAS cases (13). The other two patients in the present study (cases 2 and 3) possessed the leiomyosarcoma phenotype, expressed vimentin and diffusely expressed $\alpha$-SMA. In addition, one of the patients also expressed desmin and CD34. The results of these two patients suggest a diagnosis of leiomyosarcoma. However, the diagnosis should not be solely based on phenotype, since certain markers such as $\alpha$-SMA and desmin are not smooth muscle-specific (28). The diagnosis of leiomyosarcoma may be more accurate if it is based on the results of two examinations, compared with one examination, and should be combined with typical morphological characteristics $(23,28)$.

The ultrastructural features of intimal sarcoma include actin filaments, the presence of dense bodies in the cytoplasm surrounded by non-continuous plate bodies; these structures are similar to those observed in leiomyosarcoma (29). Regarding the genetic characteristics of PAS, a previous study using the comparative genomic hybridization method indicated that $75 \%$ (6/8 cases) of lesions are located in the 12 q13-14 region, and a number of genes were observed to be amplified (1). In addition, other less common genetic changes occurred in $3 \mathrm{p}$; losses occurred in 3q, 4q, 9p, 11q, 13q, Xp and Xq; gains occurred in 7p, 17p and 17q; and amplifications occurred in in 4q, 5p, 6p and 11q (1). Furthermore, immunohistochemistry identified intimal sarcoma in eight cases that exhibited focal expression of p53, of which, six cases overexpressed mouse double minute 2 homolog $(\mathrm{Mdm} 2)$, which suggests that the Mdm2 and p53 signaling pathways are also responsible for the pathogenesis of intimal sarcoma (1). Intimal sarcomas are, in general, malignant mesenchymal tumors with poorly differentiated fibroblasts or myofibroblasts $(23,30)$. Intimal sarcoma is not strictly a histological classification, since the term 'intimal sarcoma' is based on the sites of the lesions and the abnormal multipotency of the tumors and pleomorphic sarcomas, including leiomyosarcoma, rhabdomyosarcoma, angiosarcoma, osteosarcoma and malignant fibrous histiocytoma $(23,25,31)$. In order to improve the intervention and follow-up examinations of patients with PAS, determining the specific subtypes of intimal sarcoma, based on the classification criteria of soft tissue tumors, clinical manifestations, imaging data, histological features and immunophenotype, is extremely important $(1,2,6,13)$.

Regarding the differential diagnosis of PAS, following the exclusion of PAS from metastatic cancer, based on clinical and pathological examinations, PAS tumors must be distinguished from common tumors that occur in the heart and large vessels, such as angiosarcoma, rhabdomyosarcoma and myxoma $(1,13)$.

Angiosarcomas are mesenchymal malignancies that have differentiated from or toward vascular endothelial cells (23). Angiosarcomas are composed of spindle or epithelioid endothelial cells, which form tubular, fissures or sinus-like structures with red blood cells. The tumor cells are surrounded by reticular fibers that reveal clear vascular structures following staining. Immunohistochemically, the tumor cells express endothelial markers, including FVIII, CD31 and CD34 (23). Although one of the patients in the present study expressed CD34 (case 2), no histological differentiation was observed that would enable to confirm the diagnosis of angiosarcoma.

Rhabdomyosarcoma is rare in the cardiovascular system, and occurs more often in young people (32). The tumor cells in a rhabdomyosarcoma possess eosinophilic and granular cytoplasm, and are rich in longitudinal filaments, but not stripes. The cells are round, spindle, tadpole-, ribbon- or tennis racquet-shaped, with markedly stained nuclei.Interstitial mucus is present within the sparsely packed cells, and the tumor cells express muscle specific actin and desmin (32). In the present study, two cases expressed desmin, but the cells were mostly spindle and epithelioid, not typical of rhabdomyosarcoma.

Myxoma is a primary multipotent tumor that originates from mesenchymal cells (33). Histologically, fine reticular fibers and sparsely packed cells are often observed in rich and loose mucus. The cells have clear borders, and are star- or bipolar-shaped, but generally do not exhibit the characteristics of malignant cells, including giant cells or mitotic nuclei. The cells are positive for Alcian blue and toluidine blue staining, 
but the immunohistochemical analysis does not aid the differential diagnosis of the disease $(34,35)$.

In conclusion, PAS is an extremely rare tumor that occurs in the cardiovascular system. Due to its nonspecific clinical manifestations and radiological features, PAS is often misdiagnosed. Therefore, the diagnosis of PAS is recommended to be based on the typical morphological features and immunohistochemical analysis of the tumor tissue.

\section{References}

1. Travis WD, Brambilla E, Müller-Hermelink HK and Harris CC (eds): World Health Organization Classification of Tumours. Pathology \& Genetics of Tumours of the Lung, Pleura, Thymus and Heart. IARC Press, Lyon, 2004.

2. Bhagwat K, Hallam J, Antippa P and Larobina M: Diagnostic enigma: Primary pulmonary artery sarcoma. Interact Cardiovasc Thorac Surg 14: 342-344, 2012.

3. Mandelstamm M: Über primäre Neubildungen des Herzens. Virchows Arch Pathol Anat 245: 43-54, 1923 (In German).

4. Cox JE, Chiles C, Aquino SL, Savage P and Oaks T: Pulmonary artery sarcomas: A review of clinical and radiologic features. J Comput Assist Tomogr 21: 750-755, 1997.

5. Shehatha J, Saxena P, Clarke B, Dunning J and Konstantinov IE: Surgical management of extensive pulmonary artery sarcoma. Ann Thorac Surg 87: 1269-1271, 2009.

6. Huo L, Moran CA, Fuller GN, Gladish G and Suster S: Pulmonary artery sarcoma: A clinicopathologic and immunohistochemical study of 12 cases. Am J Clin Pathol 125: 419-424, 2006.

7. Gan HL, Zhang JQ, Huang XY and Yu W: The wall eclipsing sign on pulmonary artery computed tomography angiography is pathognomonic for pulmonary artery sarcoma. PLoS One 8: e83200, 2013.

8. Parish JM, Rosenow EC III, Swensen SJ and Crotty TB: Pulmonary artery sarcoma. Clinical features. Chest 110 1480-1488, 1996.

9. Dimitrakakis G, Zilidis G, Buchalter M and Von Oppell U: Pulmonary artery sarcoma - a challenging diagnosis: A case report. Heart Surg Forum 9: E897-E899, 2006.

10. Cook DJ, Tanser PH, Dobranowski J and Tuttle RJ: Primary pulmonary artery sarcoma mimicking pulmonary thromboembolism. Can J Cardiol 4: 393-396, 1988.

11. Etienne-Mastroianni B, Falchero L, Chalabreysse L, Loire R, Ranchère D, Souquet PJ and Cordier JF: Primary sarcomas of the lung: A clinicopathologic study of 12 cases. Lung Cancer 38: 283-289, 2002.

12. Régnard JF, Icard P, Guibert L, de Montpreville VT, Magdeleinat P and Levasseur P: Prognostic factors and results after surgical treatment of primary sarcomas of the lung. Ann Thorac Surg 68: 227-231, 1999.

13. Fletcher CDM, Unni KK and Mertens F (eds): World Health Organization Classification of Tumours. Pathology \& Genetics of Tumours of Soft Tissue and Bone. IARC Press, Lyon, 2002.

14. Bloomberg RD, Butany JW, Cusimano RJ and Leask RL: Primary cardiac sarcoma involving the pulmonary artery and valve. Can J Cardiol 19: 843-847, 2003.

15. Mussot S, Ghigna MR, Mercier O, Fabre D, Fadel E, Le Cesne A, Simonneau G and Dartevelle P: Retrospective institutional study of 31 patients treated for pulmonary artery sarcoma. Eur J Cardiothorac Surg 43: 787-793, 2013.
16. Araki Y, Tajima K, Yoshikawa M, Abe T and Suenaga Y: A case of primary pulmonary intimal sarcoma of the pulmonary artery. Nihon Kyobu Geka Gakkai Zasshi 45: 1039-1043, 1997 (In Japanese)

17. Wijesuriya S, Chandratreya L and Medford AR: Chronic pulmonary emboli and radiologic mimics on CT pulmonary angiography: A diagnostic challenge. Chest 143: 1460-1471, 2013.

18. Attinà $D$, Niro $F$, Tchouanté $P$, Mineo $G$, Russo V, Palazzini $M$, Galiè N, Fanti S, Lovato L and Zompatori M: Pulmonary artery intimal sarcoma. Problems in the differential diagnosis. Radiol Med (Torino) 118: 1259-1268, 2013.

19. Hu XP, Xu JP and Liu NN: Primary pulmonary artery sarcoma: Surgical management and differential diagnosis with pulmonary embolism and pulmonary valve stenosis. J Card Surg 24: 613-616, 2009.

20. Ebner L, Huber A, Ott D and Christe A: Pulmonary intimal sarcoma: A rare differential diagnosis for arterial filling defects on a chest CT. Acta Radiol Short Rep 3: 2047981613514052, 2014.

21. Tavora F, Miettinen M, Fanburg-Smith J, Franks TJ and Burke A: Pulmonary artery sarcoma: A histologic and follow-up study with emphasis on a subset of low-grade myofibroblastic sarcomas with a good long-term follow-up. Am J Surg Pathol 32: 1751-1761, 2008.

22. Mayer E, Kriegsmann J, Gaumann A, Kauczor HU, Dahm M, Hake U, Schmid FX and Oelert H: Surgical treatment of pulmonary artery sarcoma. J Thorac Cardiovasc Surg 121: 77-82, 2001.

23. Keel SB, Bacha E, Mark EJ, Nielsen GP, Rosenberg AE: Primary pulmonary sarcoma: a clinicopathologic study of 26 cases. Mod Pathol 12:1124-1131,1999.

24. Mattoo A, Fedullo PF, Kapelanski D and Ilowite JS: Pulmonary artery sarcoma: A case report of surgical cure and 5-year follow-up. Chest 122: 745-747, 2002.

25. Burke AP and Virmani R: Sarcomas of the great vessels. A clinicopathologic study. Cancer 71: 1761-1773, 1993.

26. Nonomura A, Kurumaya H, Kono N, Nakanuma Y, Ohta G, Terahata S, Matsubara F, Matsuda T, Asaka T and Nishino T: Primary pulmonary artery sarcoma. Report of two autopsy cases studied by immunohistochemistry and electron microscopy, and review of 110 cases reported in the literature. Acta Pathol Jpn 38: 883-896, 1988.

27. Yi CA, Lee KS, Choe YH, Han D, Kwon OJ and Kim S: Computed tomography in pulmonary artery sarcoma: Distinguishing features from pulmonary embolic disease. J Comput Assist Tomogr 28: 34-39, 2004

28. Govender D and Pillay SV: Right pulmonary artery sarcoma. Pathology 33: 243-245, 2001.

29. Croitoru AG, Klein MJ, Galla JD and Fallon JT: Primary pulmonary artery leiomyosarcoma. Cardiovasc Pathol 12: 166-169, 2003.

30. Spirtas R, Connelly RR and Tucker MA: Survival patterns for malignant mesothelioma: The SEER experience. Int J Cancer 41: 525-530, 1988

31. Burke AP and Virmani R: Osteosarcomas of the heart. Am J Surg Pathol 15: 289-295, 1991.

32. Bleisch VR and Kraus FT: Polypoid sarcoma of the pulmonary trunk: Analysis of the literature and report of a case with leptomeric organelles and ultrastructural features of rhabdomyosarcoma. Cancer 46: 314-324, 1980.

33. Jain D, Maleszewski JJ and Halushka MK: Benign cardiac tumors and tumorlike conditions. Ann Diagn Pathol 14: 215-230, 2010.

34. Burke AP and Virmani R: Cardiac my xoma. A clinicopathologic study. Am J Clin Pathol 100: 671-680, 1993.

35. Hemachandran M, Kakkar N and Khandelwal N: Giant-cell-rich myxoma of right atrium. An ultrastructural analysis. Cardiovasc Pathol 12: 287-289, 2003. 